\title{
Ionospheric Incoherent Scatter Measurements with the MU Radar: Observations of $F$-Region Electrodynamics
}

\author{
W. L. Oliver ${ }^{1 *}$, S. Fukao ${ }^{1}$, T. SATo ${ }^{1}$, T. Tsuda ${ }^{1}$, S. Kato ${ }^{1}$, I. Kimura ${ }^{2}$, A. Ito ${ }^{2}$, \\ T. SARYOU ${ }^{3}$, and T. ARAKI ${ }^{3}$ \\ ${ }^{1}$ Radio Atmospheric Science Center, Kyoto University, Uji, Kyoto 611, Japan \\ ${ }^{2}$ Department of Electrical Engineering, Kyoto University, Kyoto 606, Japan \\ ${ }^{3}$ Data Analysis Center for Geomagnetism and Spacemagnetism, Kyoto University, \\ Kyoto 606, Japan
}

(Received August 27, 1987; Revised April 25, 1988)

The MU (Middle and Upper atmosphere) radar of Japan is a 46.5-MHz, pulsemodulated, monostatic, Doppler radar with an active phased-array antenna which consists of 475 crossed Yagis. This system has been used primarily, since its initial observations with a partial system in 1983, to observe the coherent backscatter from irregularities in the troposphere, stratosphere, and mesosphere (MST radar). However, this system was also designed to be able to observe the weak incoherent scatter (IS) from the free electrons of the ionosphere. We report here the first observations of ionospheric electrodynamics using the IS capability of the MU radar. We show several examples of measurements made with one-hour time resolution for one or two days duration and then the mean behavior resulting from an average of eight experiments. We compare these results with an empirical model of electric fields derived from measurements from other incoherent scatter radars. We find good agreement between the model and the MU radar measurements for the perpendicularnorth drifts, except for the existence of some fluctuations with an 8-hour period, which we suspect will disappear from the MU mean behavior once more data are available to average. We also find good agreement between the model and measurements for the diurnal component of the perpendicular-east drifts, but the measurements additionally exhibit a semi-diurnal component of strength similar to that of the diurnal component while the model shows only a small semi-diurnal component. We also show several examples of an apparently strong control of the night-time ionospheric drift by the $F$-region dynamo mechanism, especially on the shorter time scales. Finally, we show one example of a very unusual oscillatory behavior (amplitude varying from 30 to $60 \mathrm{~m} / \mathrm{s}$, period varying from 4 to 7 hours) in the perpendicular-east velocity, while the other velocity components remained normal, during a continuous day and a half period during the GITCAD campaign of January 28-30, 1987.

*Permanent address: M.I.T. Haystack Observatory, Westford, MA 01886, U.S.A. 


\section{Introduction}

The Japanese MU (Middle and Upper atmosphere) radar is the newest of the large atmospheric radars capable of detecting the incoherent backscatter (IS) from the free electrons in the ionosphere. This radar system has been recently described by FUKAO et al. (1985a, b). This radar began operation with a partial system in 1983 and was completed in 1984. Initial observations from the middle and lower atmosphere have been published by KATO et al. (1984, 1986), among others. Ionospheric incoherent scatter observations commenced in December 1985. SATO et al. (1988) have discussed the sensitivity of the MU radar for IS detection and presented examples of typical electron density, electron and ion temperature, and ion drift velocity measurements made with this system. OLIVER et al. (1988) have discussed the MU radar electron-density and gravity-wave observations made during the strong ionospheric storm of February 6-8, 1986. In this paper, we report our initial investigations of the electrodynamic properties of the ionosphere over the MU radar. In particular, we present several examples of $F$-region drift behavior on individual days, and then we average the measurements made during the October-December 1986 period and compare these with an empirical model of electric fields derived from measurements made by other IS radars. Second, we present several examples of strong anti-correlation between the plasma drift components parallel and perpendicular to the geomagnetic field direction and interpret these in terms of the $F$-region dynamo mechanism. Last, we present an unusual and currently poorly understood observation, obtained during the GITCAD I campaign, of a day and a half of large and clear wavelike behavior in the perpendicular-east component of $F$-region drift but not in any other component.

\section{The MU Radar}

FUKAO et al. (1985a, b) have discussed the MU radar system and SATO et al. (1988) have discussed its sensitivity for IS measurements in detail. Here we give a brief summary of the characteristics of the radar of particular pertinence to the electrodynamics studies of interest in this paper. Table 1 lists several of the basic characteristics of the MU radar.

The MU radar is a monostatic radar with an active phased-array system. The antenna is a circular array of 475 crossed Yagi elements having a total diameter of 103 meters. Each antenna element is connected to a separate low-power transmit-receive module which can be driven coherently with low level pulses. Because all of the phase shifting and signal division/recombination is carried out at low power, the antenna may be phased to observe in different directions on a pulse-by-pulse basis, or up to 2500 times per second. This is a major advantage of the MU radar: its effective capability to observe in multiple directions simultaneously.

The major detriment for IS operation with the MU radar is the high system noise temperature of about $10000 \mathrm{~K}$. This is unavoidable galactic background noise 
Table 1. Basic parameters of the MU radar.

\begin{tabular}{ll}
\hline Parameter & Value \\
\hline Location & Shigaraki, Shiga, Japan \\
& \multicolumn{1}{c}{$\left(34.85^{\circ} \mathrm{N}, 136.10^{\circ} \mathrm{E}\right)$} \\
Geomagnetic parameters & Field strength: $0.0402 \mathrm{mT}$ \\
$\quad$ (300 km altitude) & Declination: $3.8^{\circ} \mathrm{W}$ \\
& Dip angle: $48.3^{\circ}$ \\
& Dip latitude: $29.3^{\circ}$ \\
Operational frequency & $46.5 \mathrm{MHz}$ \\
Antenna & Circular array of 475 crossed Yagis \\
aperature & $8330 \mathrm{~m}^{2}$ \\
steerability & $0^{\circ}-30^{\circ}$ off zenith, $5^{\circ}$ azimuth steps \\
partitioning & 25 groups of 19 elements each, \\
& each separately deriveable \\
Transmitter & 475 solid-state amplifiers \\
& $($ one for each antenna element $)$, \\
peak power & $2.4 \mathrm{~kW}$ peak $(120 \mathrm{~W}$ average $)$ power each \\
average power & $1 \mathrm{MW}$ maximum \\
pulse length & $50 \mathrm{~kW}$ maximum \\
IPP & $1-512 \mu \mathrm{s}$ \\
\hline
\end{tabular}

encountered at the MU radar operating frequency. Its effect is to degrade the signal-to-noise ratio $(S / N)$ achievable and to render the MU radar considerably less sensitive than most of the other routinely operating IS radars in the world. Nevertheless, SATO et al. (1988) have calculated that for a long-pulse $(500 \mu \mathrm{s})$ power measurement $S / N$ values in excess of unity are achievable with the MU radar, such that it can perform essentially as well as any of the other IS radars for such a measurement. On the other hand, for the very important spectral measurements, with their requirement for the transmission of a waveform of shorter pulses, it was found that the MU radar $S / N$ often drops in practice to only a few percent, such that long integration times are required to achieve acceptable statistical accuracy.

In order to achieve the best possible $S / N$ figure for ionospheric drift measurements with the MU radar, a two-pulse scheme has been implemented. This waveform consists of two $256 \mu \mathrm{s}$ pulses separated by a gap of $256 \mu \mathrm{s}$. This total of $512 \mu \mathrm{s}$ transmission time is the maximum possible during one interpulse period with the $\mathrm{MU}$ radar. This waveform yields a range resolution of $38 \mathrm{~km}$. The receiver bandwidth is matched to the pulse length $(1 / 256 \mu \mathrm{s}=4 \mathrm{kHz})$ to maximize the $S / N$ achievable. Receiver samples are collected at times $t$ and $t+512 \mu \mathrm{s}$ to provide a phase measurement of the velocity at altitude $h=t / 2 c$, where $c$ is the speed of light. Because of the length of the pulse waveform (768 $\mu \mathrm{s})$ and a system delay of $74 \mu \mathrm{s}$, the closest range which can be measured with this two-pulse experiment is $126 \mathrm{~km}$. In practice the lowest altitude measured is set to be near $200 \mathrm{~km}$ as the height resolution of the 
experiment is rather large at lower altitudes in comparison with the expected height variation of the velocity.

The two-pulse scheme provides a measure of the line-of-sight plasma drift velocity $V_{\text {los }}$ away from the radar at altitude $h$

$$
V_{\mathrm{los}}=(\lambda / 2)\left(\phi_{1}-\phi_{2}\right) / 512 \mu \mathrm{s}
$$

where $\lambda$ is the radar wavelength and $\phi_{1}$ and $\phi_{2}$ are the phases of the received signals at times $t$ and $t+512 \mu \mathrm{s}$, respectively. The phases $\phi$ are computed from the real and imaginary parts of the complex voltages

$$
v=v_{\text {real }}+i v_{\text {imaginary }}
$$

measured by the receiver detectors as

$$
\phi=v_{\text {imaginary }} / v_{\text {real }}
$$

The phase at zero lag is zero (because the IS spectrum is a purely real quantity). The lag at $512 \mu$ s occurs at a point about midway between zero lag and the lag at which the autocorrelation function (acf) magnitude first decreases to zero (this first zerocrossing is near $1 \mathrm{~ms}$ lag for usual $F$ region conditions for the MU radar operating frequency). This placement of the lag assures a sufficiently large real part of the acf for an accurate measure of $\phi$ from Eq. (3) and a sufficiently large lag separation in Eq. (1) for an accurate calculation of $V_{\text {los. }}$. From simulations considering the signal-to-noise ratio and the number of data samples collected, we estimate that the accuracy of these line-of-sight velocities is about $5-10 \mathrm{~m} / \mathrm{s}$ for average daytime electron densities and one-hour time integration (SATO et al., 1988).

The velocities reported here are uncorrected for a "transmitter frequency chirp" offset, arising from a slight droop in transmitter voltage and a resultant phase lag between the two transmitted pulses. An initial measurement of this chirp velocity has given a value of $22.8 \mathrm{~m} / \mathrm{s}$ for the line-of-sight velocity bias. For a truly stationary ionosphere this bias gives an apparent $22.8 \mathrm{~m} / \mathrm{s}$ drift toward the radar. Upon computation of components in the geomagnetic field reference frame, we find a 18.1 $\mathrm{m} / \mathrm{s}$ effect on the parallel velocity, a $16.2 \mathrm{~m} / \mathrm{s}$ effect on the perpendicular-north velocity, and no effect on the perpendicular-east velocity (these values apply for the usual two-pulse observing directions given in the following section). The stability of this chirp value has not been verified, however, and in the current report we have chosen not to correct our displayed velocities for the chirp effect. This will not affect our main thrust of interpreting the diurnal variation of the electric field components, however.

The two-pulse drift velocity measurement is usually alternated with a power profile measurement during MU radar IS experiments. The power measurement usually employs a 7-bit Barker-coded pulse with a $64 \mu$ s sub-pulse length, providing a $9.6 \mathrm{~km}$ range resolution. This measurement is made throughout the $E$ and $F$ regions. 
The $E$-region signals are often contaminated by meteor echoes (SATO et al., 1988). The $F$-region signal usually becomes too weak to be useful beyond about $600 \mathrm{~km}$ altitude. Normally the velocity measurement is run for 45 consecutive minutes and the power measurement for the remaining 15 minutes of each hour, with data being written to tape about every half minute.

The received power $P_{\mathrm{r}}$ is related to the electron density $N_{\mathrm{e}}$ as

$$
P_{\mathrm{r}}=C N_{\mathrm{e}} / R^{2}\left(1+T_{r}\right)
$$

where $C$ is a lumped radar system constant, $R$ is the range to the scattering volume, and $T_{\mathrm{r}}$ is the ratio of the electron to the ion temperature (SATO et al., 1988). We normalize the measured $R^{2} P_{\text {r }}$ profile at its peak to the $F$-layer peak density as measured simultaneously by an on-site ionosonde to derive the MU radar " $N_{\mathrm{e}}$ " profile. This profile is accurate at the $F$-layer peak $h_{\max }$ but at any other height $h$ is in error by the ratio $\left[1+T_{\mathrm{r}}\left(h_{\max }\right)\right] /\left[1+T_{\mathrm{r}}(h)\right]$. In the worst case this could lead to a factor of two error in our $N_{\mathrm{e}}$ estimate.

\section{Electric Field Patterns}

To introduce the ionospheric electric field patterns observed above the MU radar, we present here the results of three experiments ranging from 29 to 38 hours duration during October 7-8, November 6-8, and December 16-18, 1986 and five night-time-only experiments (October 27-30 and December 2-3, 1986). These experiments consisted of a one-hour experiment cycle with power-profile data being collected for 15 consecutive minutes and two-pulse drift velocity data being collected for the remaining 45 minutes of each cycle. Exceptions to this pattern occurred during the November experiment, when no power data were acquired before 17 LT on November 7 (some time gaps also existed during the November experiment), and during the long December experiment, when power data were continued to be acquired another 30 hours after termination of the velocity experiments. For the large-scale temporal patterns of primary interest in this paper, we shall show the power and velocity results post-integrated to 15 and 45 minutes, respectively. These experiments were carried out in four observing positions, magnetic north, east, south, and west, all at a $20^{\circ}$ antenna zenith angle, to allow the vector velocity to be reconstructed. We will show the velocities averaged over the height range 217-303 km, i.e., centered on $260 \mathrm{~km}$ altitude, near the $F$-layer peak.

Figures 1 and 2 show the velocities and densities measured during the three full-day experiments mentioned above. The velocities have been resolved into magnetic coordinates, with components parallel, perpendicular north, and perpendicular east with respect to the magnetic field direction. We will first comment on the behavior observed on these days insofar as their individual behaviors are of particular interest; then we will concentrate our emphasis on the patterns obtained by averaging all eight periods.

Concerning the individual day plots, we can see that there is much day-to-day 

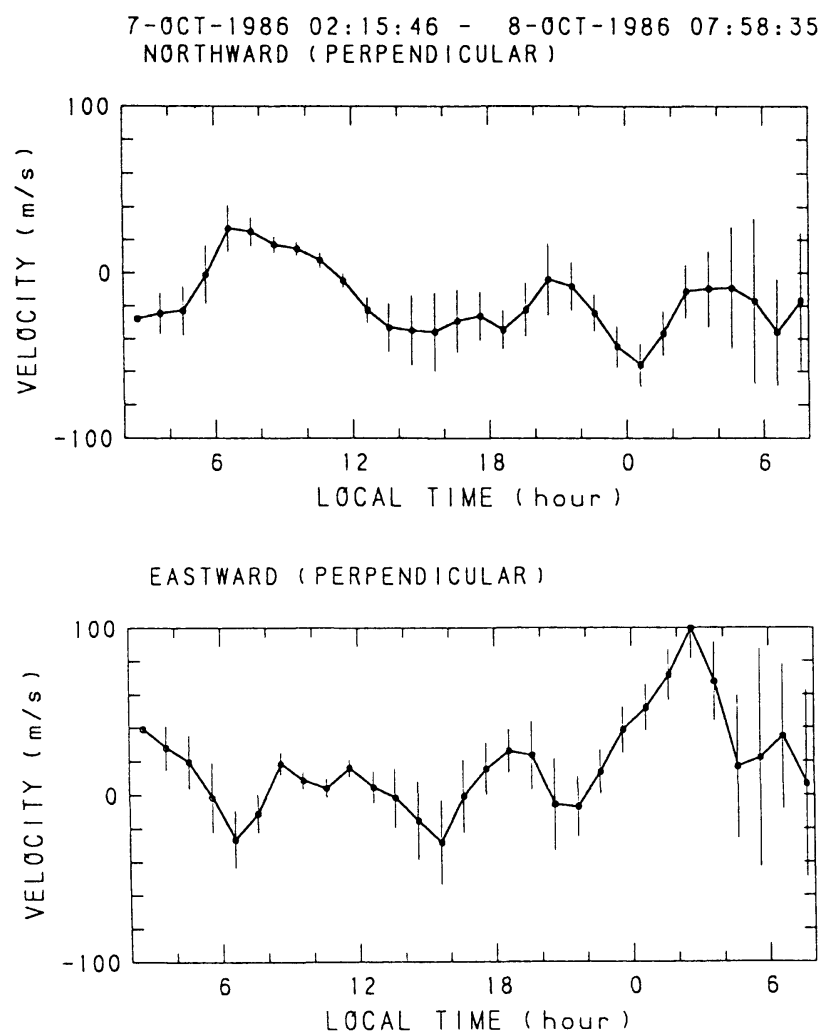

UPWARD (PARALLEL)

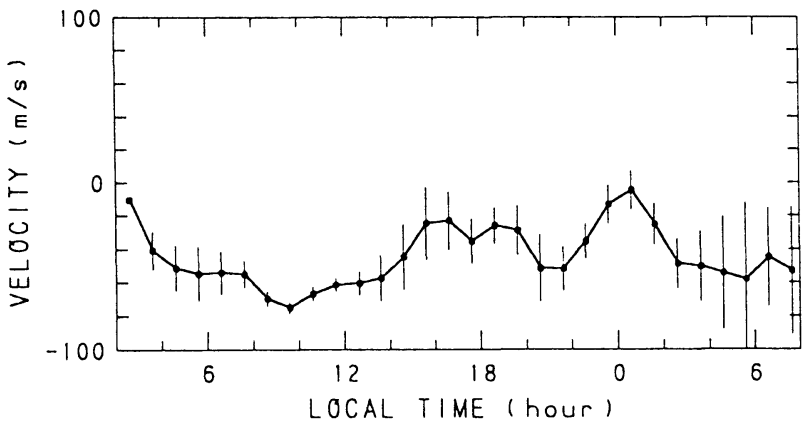

Fig. 1(a). Ionosphere drift velocity averaged over $217-303 \mathrm{~km}$ altitude measured by the $\mathrm{MU}$ radar during October 7-8, 1986. 

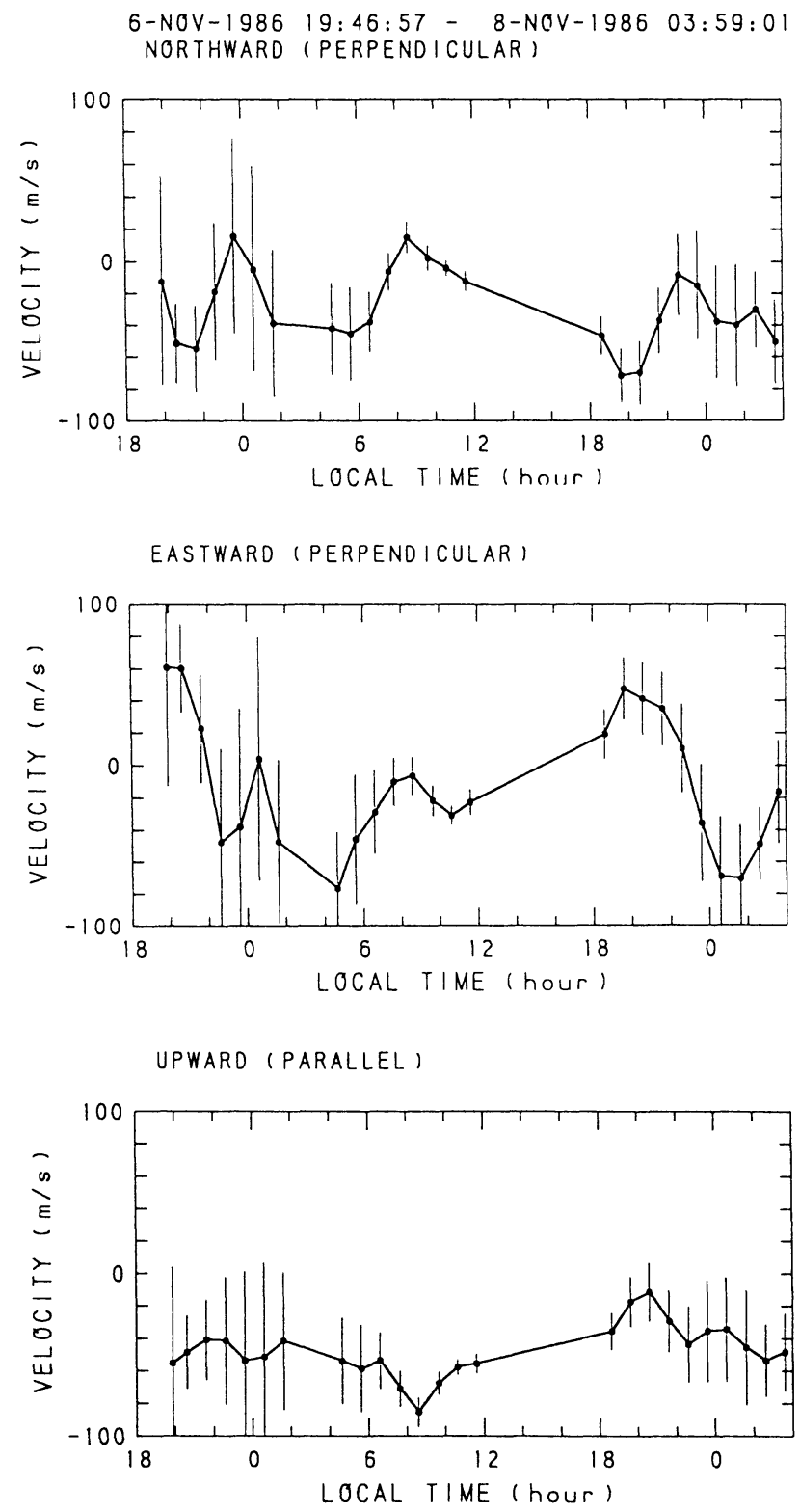

Fig. 1(b). Ionosphere drift velocity averaged over $217-303 \mathrm{~km}$ altitude measured by the $\mathrm{MU}$ radar during November 6-8, 1986. 

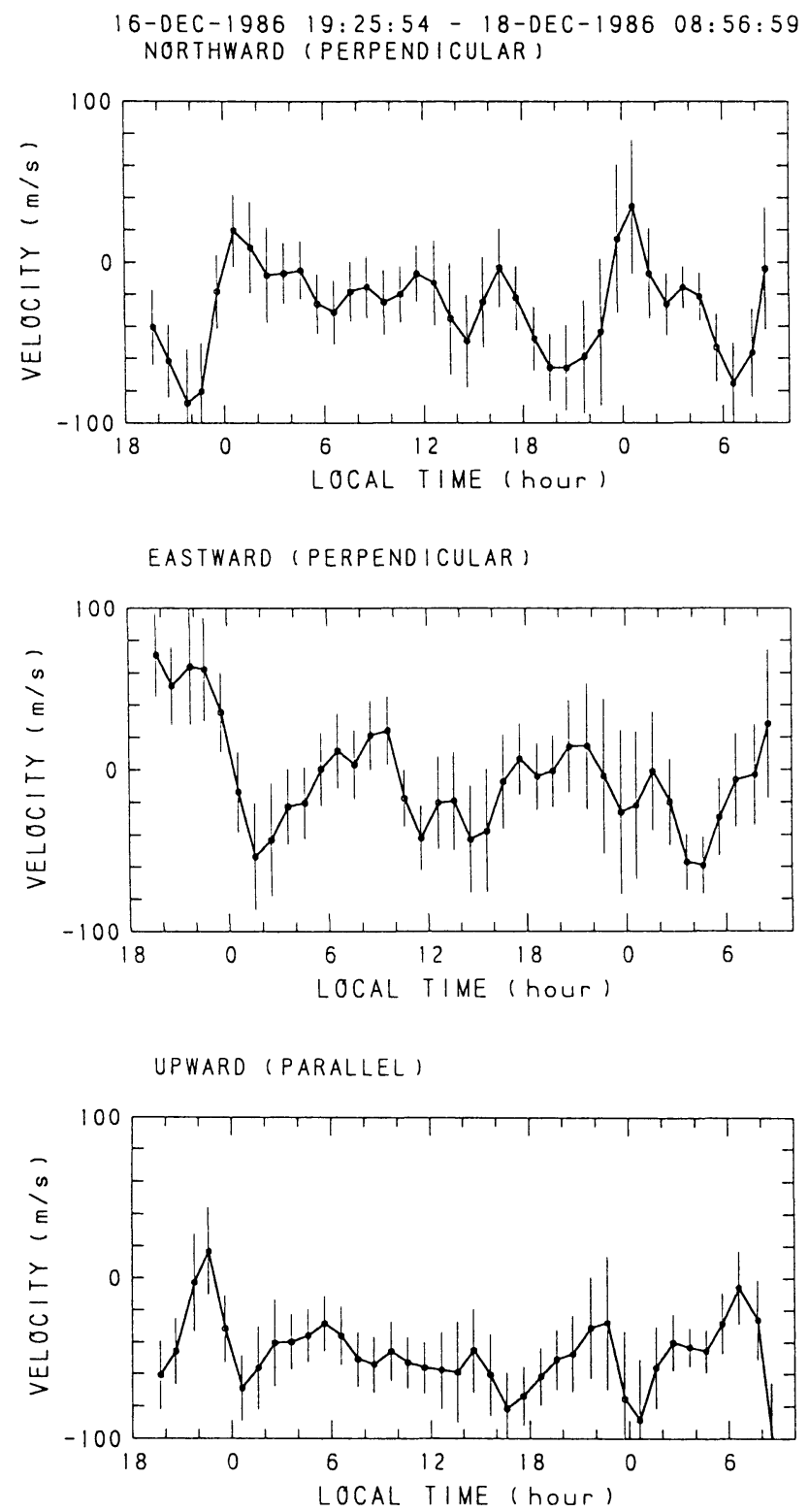

Fig. 1(c). Ionosphere drift velocity averaged over $217-303 \mathrm{~km}$ altitude measured by the MU radar durir December 16-18, 1986. 


$$
\begin{array}{rrr}
7-0 C T-198601: 59: 43-8-0 C T-1986 & 05: 59: 41 \\
& \text { IOLOG( } \mathrm{Ne})\left(\mathrm{cm}^{-3}\right)
\end{array}
$$
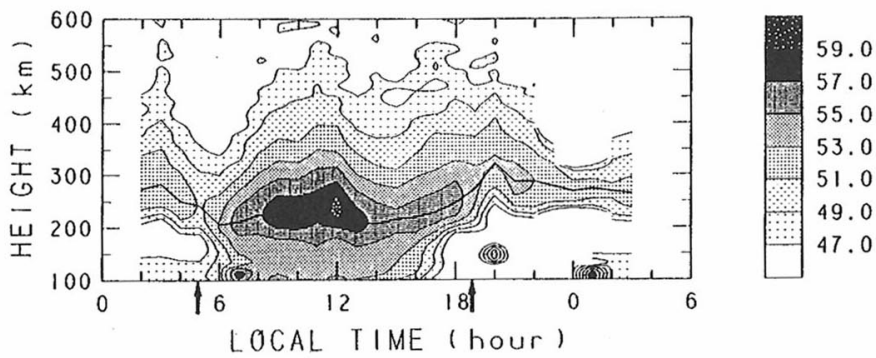

(a)

$$
\begin{array}{rrr}
7-N O V-1986 \quad 17: 52: 56-8-N O V-1986 & 03: 59: 36 \\
& 10 L O G(\mathrm{Ne})\left(\mathrm{cm}^{-3}\right)
\end{array}
$$
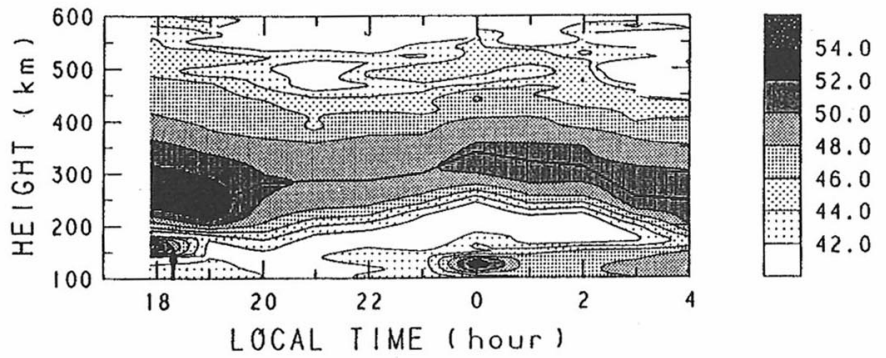

(b)

$$
\begin{array}{rrr}
16-D E C-1986 \quad 19: 14: 02-19-D E C-1986 & 14: 59: 45 \\
& 10 L O G(\mathrm{Ne})\left(\mathrm{cm}^{-3}\right)
\end{array}
$$
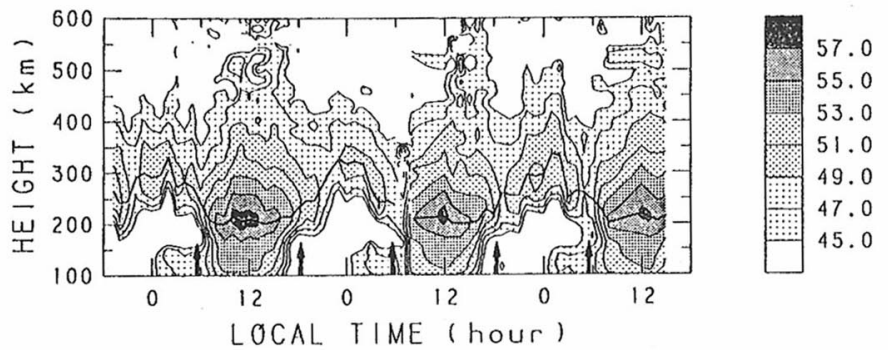

(c)

Fig. 2. (a) Ionosphere density measured by the MU radar during October 7-8, 1986. The solid line superposed upon the contour plot represents the height of the $F$-layer peak. (b) Ionosphere density measured by the MU radar during November 7-8, 1986. The solid line superposed upon the contour plot represents the height of the $F$-layer peak. (c) Ionosphere density measured by the MU radar during December 16-19, 1986. The solid line superposed upon the contour plot represents the height of the $F$-layer peak. 
variability. This result is expected on the basis that, at the relatively low latitude of Shigaraki, the electric fields driving the perpendicular ion motions are caused by neutral winds of a tidal origin in the dynamo $(E)$ region, and these winds are known to be quite variable (RICHMOND et al., 1976). Another point evident from close inspection of the drifts is that the parallel and perpendicular-north components are often anticorrelated, particularly at night, and especially on the shorter time scales. We attribute this effect to the $F$-region dynamo mechanism, which we consider more thoroughly in a later section of this paper.

Figures 3 and 4 show the average velocity and density behaviors obtained by averaging all eight experiments from the October-December 1986 period. Figure 5 shows dial plots of the amplitudes and phases of harmonic components fitted to these averaged data. First, we will discuss the parallel drifts. On average, these drifts are more strongly downward during the day and less strongly downward at night. Parallel drifts in the ionosphere are caused by two processes: neutral wind forcing and diffusion. Winds may blow the ionization up or down the field lines while the diffusion velocity is always downward, more strongly downward below the $F$-layer peak and less strongly downward above it. The $260-\mathrm{km}$ mean height to which these results apply lies above the $F$-layer peak height during the day and below it at night (Fig. 4), such that we would expect the drift component due to diffusion to be more strongly downward at night; this is in direct opposition to our observation. Thus we must attribute the observed diurnal variation of the parallel velocity to a poleward wind by day and an equatorward wind by night. This is consistent with our basic concept of $F$ region winds blowing in great circle paths around the globe from the dayside pressure bulge caused by direct, in situ solar heating.

The perpendicular drifts show a more complex behavior than the parallel drifts. The eastward component shows a decidedly semi-diurnal pattern while the northward component seems to exhibit periodic components with even shorter periods. We can clearly see evidence of fluctuations of about an 8-hour period in the data for individual days, and this effect is also reflected in the average pattern. We wish to recall, however, that in the first evaluation of perpendicular drifts measured by the French IS radar (BLANC et al., 1977) from a limited set of observations it was concluded that one of the perpendicular components was dominated by a ter-diurnal component, while a later evaluation from a much larger data set (BLANC and AMAYENC, 1979) concluded that the semi-diurnal component was dominant. We similarly question if the ter-diurnal appearance of our perpendicular-north drifts represents a permanent feature of behavior, suspecting rather that it owes its presence to the greater variability of the nighttime drifts and the lack of a sufficient data base to integrate out its random occurrence.

We now wish to compare these initial mean behaviors of the MU radar perpendicular drifts with the empirical electric field model of RICHMOND et al. (1980). Richmond collected ionospheric drift data from four incoherent scatter facilities and fit a smooth analytic model to them to represent the mean variations as a function of magnetic latitude, magnetic local time, universal time, and day of year. While all of these variations were significant, the latitude and local-time variations were found to 
NORTHWARD ( PERPENDICULAR)

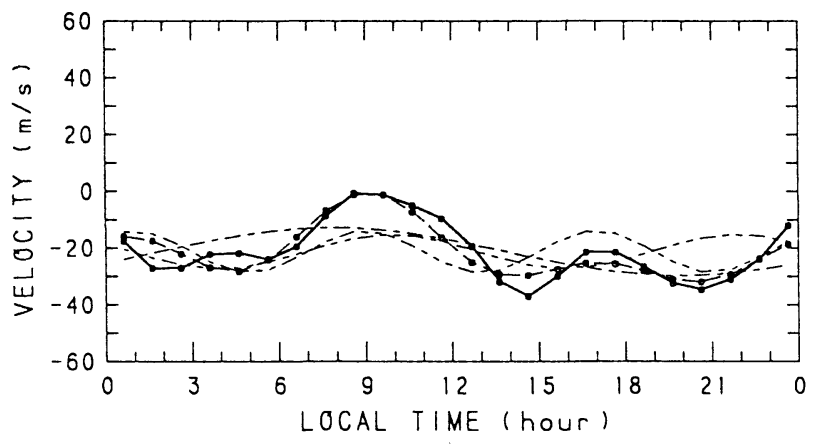

EASTWARD (PERPEND ICULAR)

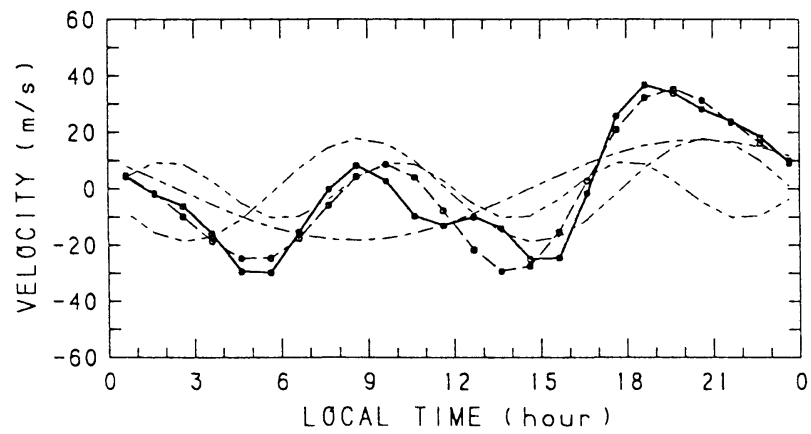

UPWARD (PARALLEL)

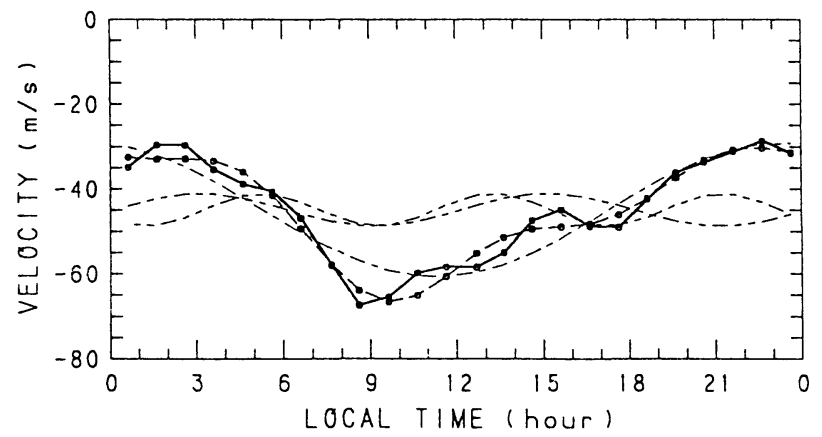

Fig. 3. Mean ionosphere drift velocity averaged over $217-303 \mathrm{~km}$ altitude measured by the MU radar during October-December 1986. The solid line represents the averaged data. Dashed lines without symbols represent the 24-, 12-, and 8-hour period harmonic components of the data while dashed lines with symbols represent the sum of the daily mean and the three diurnal harmonics. 
$10 \operatorname{LOG}(\mathrm{Ne})\left(\mathrm{cm}^{-3}\right)$
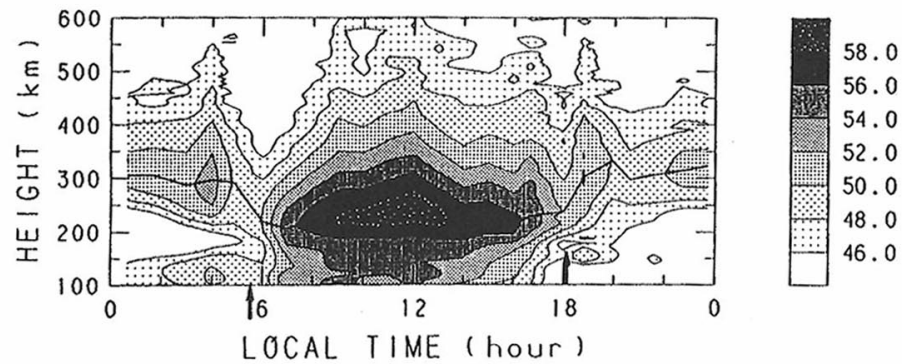

Fig. 4. Mean ionosphere density measured by the MU radar during October-December 1986. The solid line superposed upon the contour plot represents the height of the $F$-layer peak.

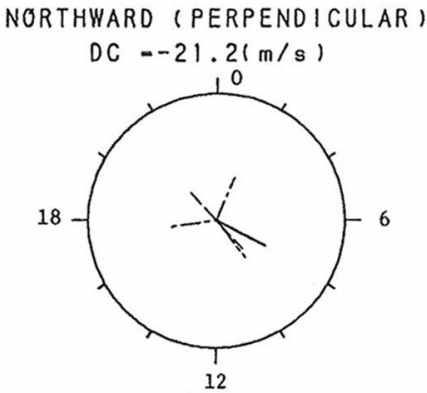

LOCAL TIME OF MAXIMUM

UPWARD (PARALLEL)

DC $-44.9(\mathrm{~m} / \mathrm{s})$

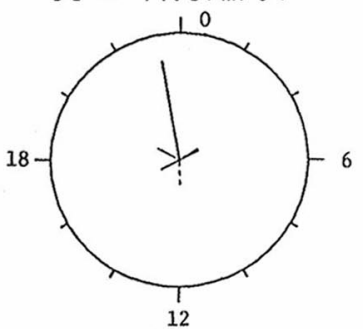

LOCAL TIME OF MAXIMUM

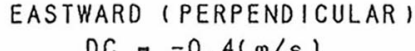

$D C--0.4(\mathrm{~m} / \mathrm{s})$

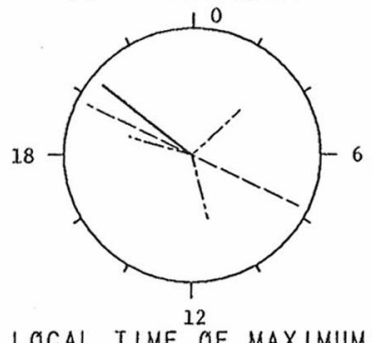

LOCAL TIME OF MAXIMUM

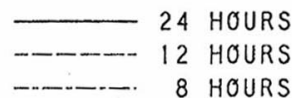

MAXIMUM AMPLITUDE $=20.0(\mathrm{~m} / \mathrm{s})$

HEIGHT RANGE : $217.2-302.9(\mathrm{~km})$

Fig. 5. Dial plots summarizing the harmonic information from Fig. 3. Given are the daily mean and the amplitude and phase of the three diurnal harmonics for the three velocity components. 
be the predominant ones, giving the main pattern of behavior. We will make our comparison, therefore, with Richmond's UT and seasonally averaged model, as shown in Fig. 6. The $30^{\circ}$ magnetic latitude curve is the one of interest for our comparison.

Looking first at Richmond's model, in the perpendicular-north drifts we see a predominantly semi-diurnal pattern, with a major peak in the northward flow near 10 LT and a minor peak near $22 \mathrm{LT}$, and a major peak in the southward flow near $18 \mathrm{LT}$ and a minor peak near $2 \mathrm{LT}$. The perpendicular-east velocity is decidedly diurnal in nature, with an eastward peak near 19 LT and a westward peak near 10 LT. Looking now at the MU radar perpendicular-north velocities, we see strong similarities with Richmond's model. The MU data show a large northward peak near 9 LT and a minor northward peak near $24 \mathrm{LT}$. We also observe the minor peak in southward velocity near 2 LT, but the major southward peak expected at $18 \mathrm{LT}$ is masked by a northward perturbation having a duration of about 8 hours. As we discussed earlier, we suspect that this 8-hour feature may not persist in the average MU radar behavior when we are able to accumulate a larger data base.

There are some very large differences between Richmond's model perpendiculareast drifts and the MU radar average behavior, however. The model of Richmond, and the radar data from the four IS stations from which the model was derived, all

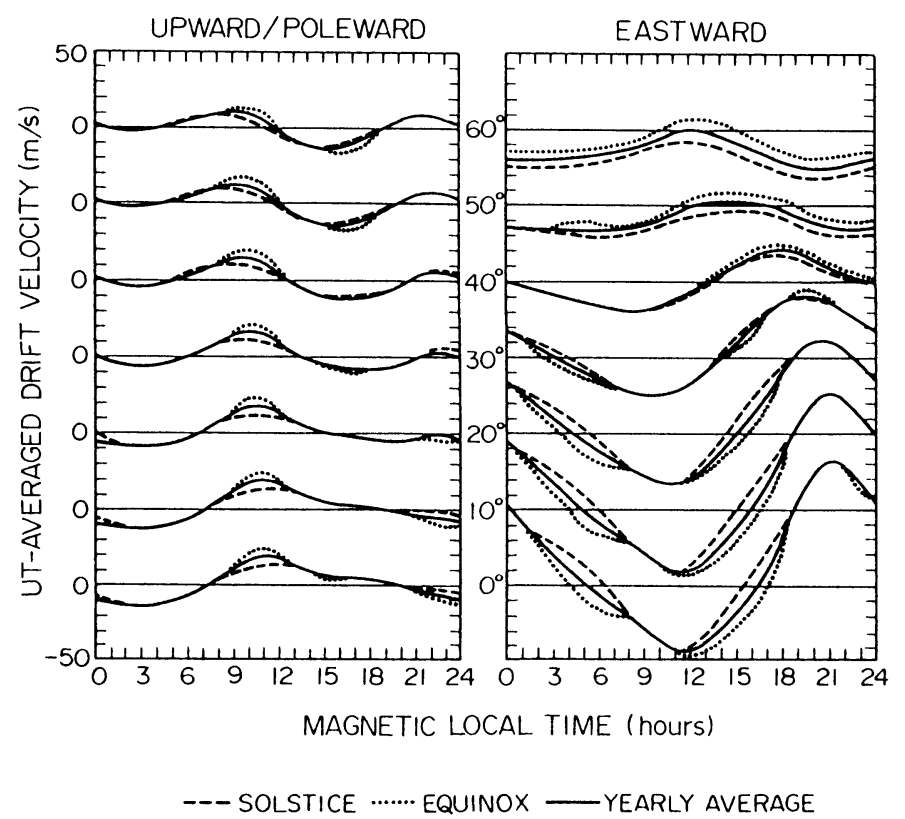

Fig. 6. UT-averaged model drifts as a function of magnetic local time at spacings of $10^{\circ}$ magnetic latitude for solstice (dashed curves), equinox (dotted curves), yearly average (solid curves) conditions (RICHMOND et al., 1980). 
show definite diurnal patterns while the MU radar data contains an equally strong semi-diurnal component.

RICHMOND et al. (1976) found that almost all of the quiet-day ionospheric currents at mid and low latitudes could be attributed to a combination of an in-situgenerated diurnal tide and an upward-propagating semi-diurnal tide. The diurnal tide should be stable from day to day, owing to the stability of the solar illumination, while the electric fields generated by the semi-diurnal tide are known to be highly variable, owing to the variability of the winds generating them. The effectiveness of these two tidal components varies with latitude, with the semi-diurnal component gaining increasing importance toward high latitudes for the perpendicular-east drift (Fig. 6). The MU drifts similarly seem to have gained a substantial semi-diurnal component in the perpendicular east component. The diurnal component of the MU perpendiculareast drifts shows excellent phase agreement with Richmond's model (maximum eastward drift near 20 LT), consistent with its being a stable, in-situ-generated tide. The presence of the large semi-diurnal component, however, indicates that this component is much more effectively generated at the MU radar longitude than at the longitudes of the radars contributing data to Richmond's model. Richmond did not have sufficient data to determine longitudinal variations for his model. Also, he reported difficulty in achieving a good fit to the Millstone Hill and Saint Santin (the two most poleward stations) data sets simultaneously owing to the totally different behavior in their east-west components. GONZALES et al. (1978) have found that the electric-field behavior at Millstone Hill and Saint Santin are similar only under very quiet conditions. At other times they suggest that auroral fields play an important role in the behavior at the Millstone Hill location owing to its proximity to the auroral region. For the MU radar location we find yet another pattern of behavior in the perpendicular-east drifts. This component seems to be quite variable with global location, and its generation mechanism needs to be studied more thoroughly.

\section{F Region Dynamo Observations}

We often observe the ionospheric plasma to drift in a largely horizontal fashion over the MU radar at night. Examples of this behavior are shown in Figs. 7 and 8, which show the plasma drift components and electron density profile behavior measured during two experiments (other examples of this behavior may be seen in Figs. 1, 3, and 10). We see here that there is a very strong anti-correlation between the parallel and perpendicular-north ion drift components at night, particularly on the shorter time scales. Neither the vertical velocity component ("upward component" in Figs. 7 and 8) nor the $F$-layer peak height participate in the fluctuations seen in the perpendicular-north and parallel velocity components.

Although the vertical velocity in these cases is small, the vertical projections of the drift components parallel and perpendicular to the magnetic field direction are not necessarily small but rather are observed largely to cancel each other. We interpret this to be a self-cancelling reaction generated within the upper-atmosphere/ ionosphere system by the drag forces operating when the neutral and ionized gases 
attempt to move through each other. This reaction may occur whether the initial forcing agent is a neutral wind or an electric field.

If the initial agent is an electric field, the initial plasma motion perpendicular to the magnetic field lines will force, through collisions with the neutrals, a steady-state neutral wind motion horizontally along the same directional bearing. This neutral wind will then force, through collisions with the ions, a plasma motion along the field lines, but with a vertical component directed oppositely to the vertical component of the original perpendicular motion driven by the electric field. These oppositely directed vertical components of flow cancel to produce a steady-state horizontal reaction of the ionosphere to the impression of an electric field (see RISHBETH and GARRIOTT (1969) and references cited therein).

On the other hand, if the initial forcing agent is a neutral wind, the collisions of the neutrals with the ions will create an $F$-region dynamo (RISHBETH, 1971) electric field which, under certain conditions, will again cause the plasma to move horizontally. This effect is generated as follows. Because the gyrofrequencies of the ions and electrons are much greater than their collision frequencies, the ions and electrons are pushed largely parallel along the magnetic field lines by neutral winds in the $F$ region. Nevertheless, the collisions do force a small component of both ion and electron motion across the field lines; the cross-field electron flow is negligible compared to the cross-field ion flow because of the much smaller collisionfrequency/gyrofrequency ratio for the electrons. Thus a current is driven in the $F$ region. The circuit for this current is completed along the magnetic field lines (which are good conductors) and through the $E$ region, where the familiar $E$-region dynamo process allows a current to flow easily. At night, however, the $E$-region electron density, and hence its electrical conductivity, is usually too low to carry the full current produced in the $F$ region, and the current flow in the $F$ region then builds up a space-charge electric field. This electric field forces a vertical component of ion motion which opposes the vertical component of ion motion parallel to the magnetic field lines caused by the initial wind. If the current path is completely broken, then the electric field will attain the magnitude necessary to result in total cancellation of the vertical ion motion, and a horizontal plasma flow will result.

The means of distinguishing between these two processes lies in their time constants. The time constant is inversely proportional to the collision frequency of the gas being forced into motion, or, equivalently, to the number density of the gas doing the forcing. BARON and WAND (1983) have shown that the time constant for the ions to set the neutrals into motion is about 38 minutes for an ion concentration of $10^{12} \mathrm{~m}^{-3}$ but 6.25 hours for a concentration of $10^{11} \mathrm{~m}^{-3}$. Because the neutral concentration is several orders of magnitude larger than the ion concentration, the time constant for the neutrals to set the ions into motion is on the order of seconds. It is our observation of essentially instantaneous correlation between the parallel and perpendicular components of the ion drift that leads us to ascribe the $F$-region dynamo process as the cause of the predominantly horizontal plasma flow seen at nighttime over the MU radar.

The perpendicular-north and parallel drifts should be in the ratio $r=\sin I /$ 

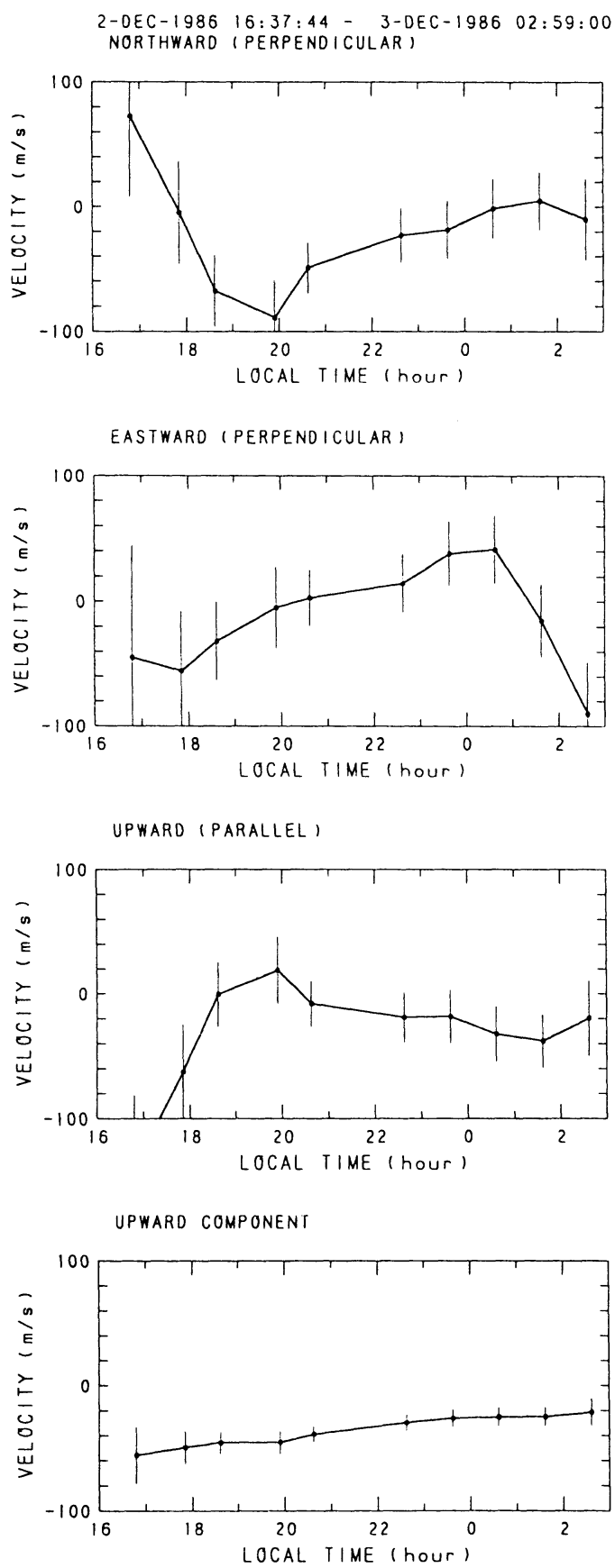

Fig. 7(a). Ionosphere drift velocity averaged over $217-303 \mathrm{~km}$ altitude measured by the MU radar during December 2-3, 1986 

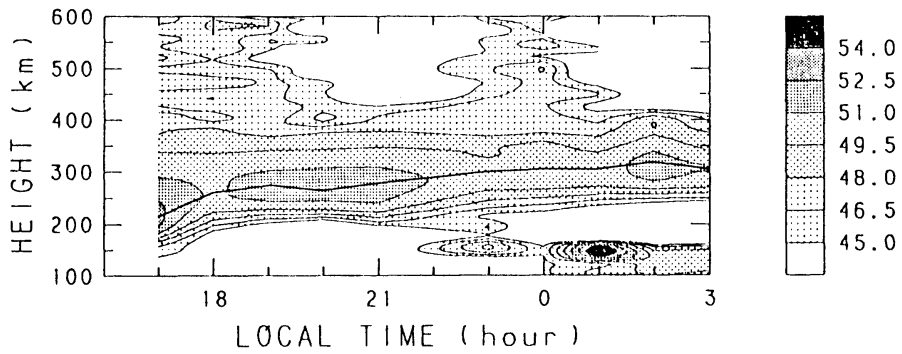

Fig. 7(b). Ionosphere density measured by the MU radar during December 2-3, 1986. The solid line superposed upon the contour plot represents the height of the $F$-layer peak.

$\cos I=1.12$ ( $I$ is the magnetic field dip angle - see Table 1$)$ if they are to cancel each other. We have computed the ratio of the change in perpendicular-north velocity to the change in parallel velocity (recall that we have some uncertainty in the dc bias of our drift measurements) for each neighboring pair of measurements in Figs. 7(a) and $8(\mathrm{a})$, with the result that, on average, the ratio $r$ was 1.33 and 1.40 for these two cases. This relates to the shorter-term (hour scale) variations. If the dynamo circuit was not completely broken by low $E$-region densities, we would expect a partial shorting of the dynamo electric field and consequently a ratio $r$ less than 1.12 . We have unexpectedly achieved values somewhat greater than 1.12 .

If the $F$-region dynamo electric field were to fully cancel the wind-induced vertical drifts of the ionosphere, then the residual vertical velocity (the bottom frames in Figs. 7(a) and 8(a)) should be due to diffusion. Following our previous discussion, this drift should be always downward but more strongly downward when the altitude of observation is below the $F$-layer peak height and less strongly downward when it is above the peak. This would appear as a negative correlation between the vertical velocity and $h_{\max }$ in comparing Fig. 7(a) with 7(b) or 8(a) with 8(b). While the changes in these parameters are small, we see, in fact, a generally positive correlation, and so we must look elsewhere for causes of this residual motion. This could be due to an additional electric-field (perhaps of tidal origin) lifting mechanism (the low nighttime ion density should prevent effective ion-drag forcing of a compensating neutral wind) or to a vertical neutral wind, which would create a dynamo effect carrying the plasma vertically with it.

The anti-correlation between the upward-parallel and perpendicular-north ionosphere drift velocities has been observed with other IS radars, e.g., BEHNKE and HARPER (1973) and BEHNKE et al. (1985) at Arecibo and TAYLOR (1974) at Malvern, and RISHBETH $(1979,1981)$ has reviewed the evidence on these drift components. Behnke et al. have shown that either the $F$-region dynamo or an external-electricfield/ion-diffusion mechanism can control the drift at Arecibo. The latter mechanism, 

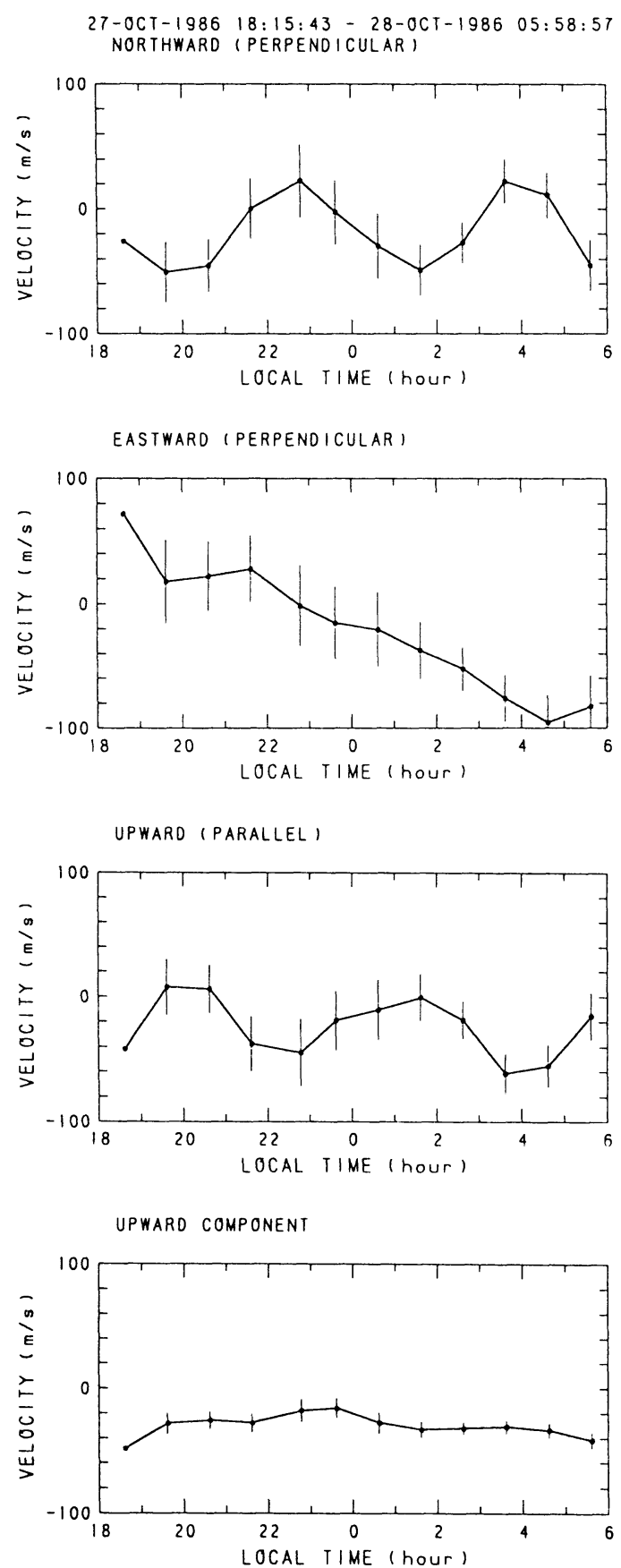

Fig. 8(a). Ionosphere drift velocity averaged over $217-303 \mathrm{~km}$ altitude measured by the MU radar during October 27-28, 1986. 


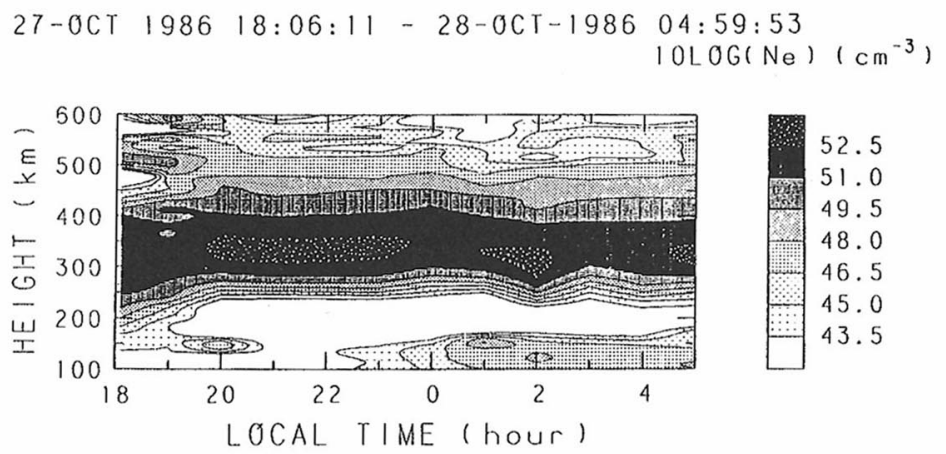

Fig. 8(b). Ionosphere density measured by the MU radar during October 27-28, 1986. The solid line superposed upon the contour plot represents the height of the $F$-layer peak.

consisting of a strong raising of the $F$-layer by electric fields followed by plasma diffusive flow down the field lines, cannot be a cause of the horizontal plasma flows shown in this paper as the $F$-layer is observed to have only modest movement. In our limited set of MU radar observations, we often, but not always, see the velocity anti-correlation reported here. Neither can we rule out the possibility that the anticorrelation, when seen, is sometimes caused by other mechanisms, as mentioned above and by the authors referenced (ion drag, magnetospheric electric-field penetration, conjugate effects, external-electric-field/ion-diffusion).

\section{GITCAD I Preliminary Observations}

With the commencement of IS operations in December 1985, the MU radar began quasi-monthly observations coordinated with the other IS radars of the world according to the schedule established on the International Geophysical Calendar. In this section we wish to show preliminary MU radar observations made during the special GITCAD (Global Ionosphere Thermosphere Coupling And Dynamics) campaign of January 1987.

Figure 9 shows the electron density measurements made during this period. The behavior is rather normal and diurnally repetitive. Once again, the apparently elevated densities in the $E$ region at night probably result from contamination by meteor echoes. Figure 10 shows the plasma drift velocity components in the geomagnetic field reference frame. The striking characteristic of these velocities is the large-amplitude, long-duration, periodic nature of the perpendicular-east component from about 4 LT on January 29 until the end of the experiment, and also the continuous, monotonic, slow change in the mean background drift from eastward flow to westward flow throughout the two-day duration of the experiment, amounting to a change of some $120 \mathrm{~m} / \mathrm{s}$ throughout the period. These data show three full cycles of a $\pm 60-\mathrm{m} / \mathrm{s}$ amplitude oscillation from about 4 LT to 24 LT on January 

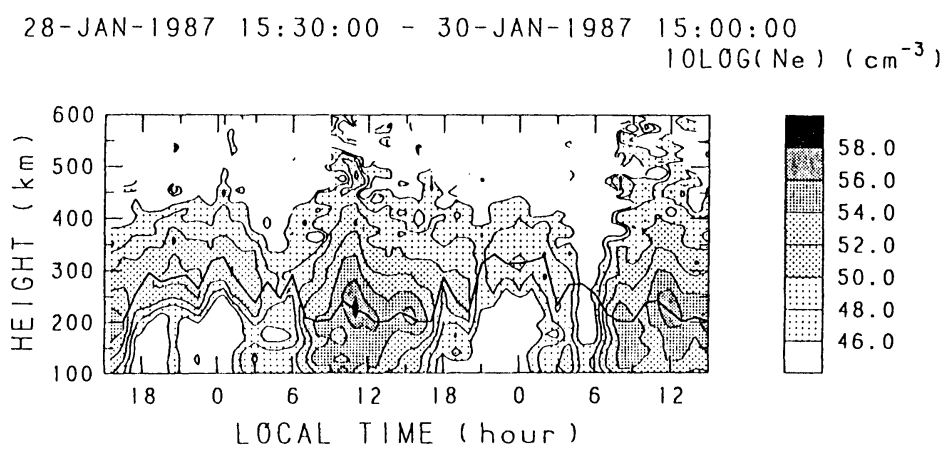

Fig. 9. Ionosphere density measured by the MU radar during the GITCAD campaign of January 28-30, 1987. The solid line superposed upon the contour plot represents the height of the $F$-layer peak.

29 , followed by four cycles of a $\pm 30-\mathrm{m} / \mathrm{s}$ amplitude oscillation from $0 \mathrm{LT}$ to $16 \mathrm{LT}$ on January 30. These behaviors are completely absent in the perpendicular-north component. The parallel component shows a small-amplitude periodicity, with some apparent short-term correlation with the eastward velocity perturbations, but over the longer term this correlation is poor. The shorter-time-scale fluctuations in the parallel and perpendicular-north velocities are anticorrelated at night, again a probable $F$-region dynamo effect. We have inspected solar and geomagnetic data for this period as available at the time of writing this report, and find this January period to be remarkably devoid of interesting events. Minor flare and storm commencements and the times of small enhancements in $K p$ seem uncorrelated with the timing of our oscillatory perturbations.

The authors are unaware of any other report in the literature of electric field behavior of the nature seen by the MU radar during the GITCAD experiment of January 1987. Further consideration of the cause of this extraordinary behavior must await the availability of magnetometer measurements.

\section{Summary}

The MU radar at Shigaraki, Japan began operation as an ionospheric incoherent scatter (IS) radar in December 1985 and is now contributing to the study of ionospheric electrodynamics. In this paper we have shown several examples of one- or two-day plasma drift velocity experiments with one-hour time resolution, and we have averaged eight experiments from October to December 1986 to derive an initial estimate of the mean electric field behavior for the radar location. Comparison with the electric field model of RICHMOND et al. (1980), which was derived from collections of data from four low- and mid-latitude IS radars, showed the following results. The MU radar perpendicular-north drifts showed good agreement with the model, with the exception of the appearance of a fluctuation of 8-hour period in the MU radar 

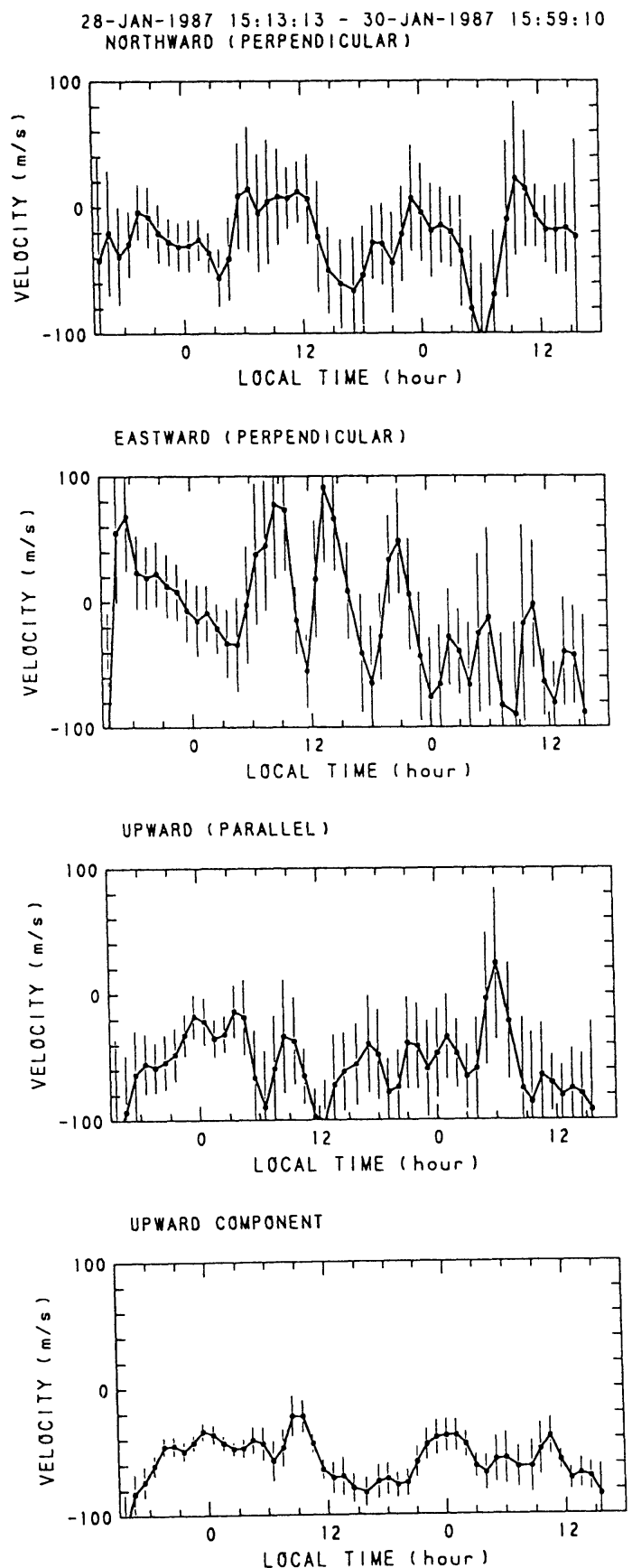

Fig. 10. Ionosphere drift velocity averaged over $217-303 \mathrm{~km}$ altitude measured by the MU radar during the GITCAD campaign of January 28-30, 1987. 
data; we suspect that this 8-hour feature of the MU mean behavior may disappear when we have more data to include in our average. The MU radar perpendicular-east drifts, however, showed a very basic difference from the model: while the diurnal components of the data and model agreed well, the data showed a large semi-diurnal component while the model had only a small one.

We have also shown several examples of highly anti-correlated behavior between the parallel and perpendicular-north components of the plasma velocity at night, especially on the shorter time scales. Because this correlation occurs with essentially no measurable lag time, we attribute this behavior to the $F$-region dynamo mechanism.

Last, we have shown a case of highly unusual drift behavior occurring during the GITCAD campaign of January 28-30, 1987. Seen in the perpendicular-east velocity component, but in no other component, was a day and a half of oscillatory behavior with amplitudes varying from 30 to $60 \mathrm{~m} / \mathrm{s}$ and period from 4 to 7 hours, all superposed on a background mean wind which changed linearly from east to west over the 2-day duration of the experiment, amounting to a $120-\mathrm{m} / \mathrm{s}$ change in the mean drift over that period.

The authors are indebted to the staff of the Radio Atmospheric Science Center and the Department of Electrical Engineering of Kyoto University for their helpful support. One of us (WLO) was supported for this work as a Visiting Research Scholar at Kyoto University and as a Visiting Associate Professor at the Radio Atmospheric Science Center. The MU radar belongs to and is operated by the Radio Atmospheric Science Center of Kyoto University.

\section{REFERENCES}

BARON, M. J. and R. H. WAND, $F$ region ion temperature enhancements resulting from Joule heating, $J$. Geophys. Res., 88, 4114-4118, 1983.

BeHNKE, R. A. and R. M. HARPER, Vector measurements of $F$ region ion transport at Arecibo, J. Geophys. Res., 78, 8222-8234, 1973.

Behnke, R., M. Kelley, C. Gonzales, and M. Larsen, Dynamics of the Arecibo ionosphere, J. Geophys. Res., 90, 4448-4452, 1985.

Blanc, M. and P. AmAYenc, Seasonal variations of the ionospheric $\boldsymbol{E} \times \boldsymbol{B}$ drifts above Saint-Santin on quiet days, J. Geophys. Res., 84, 2691-2704, 1979.

Blanc, M., P. Amayenc, and C. TAieb, Electric field induced drifts from the French incoherent scatter facility, J. Geophys. Res., 82, 87-97, 1977.

Fukao, S., T. Sato, T. Tsuda, S. Kato, K. Wakasugi, and T. Makihara, The MU radar with an active phased array system, 1. Antenna and power amplifiers, Radio Sci., 20, 1155-1168, 1985a.

Fukao, S., T. Tsuda, T. Sato, S. Kato, K. Wakasugi, and T. Makihara, The MU radar with an active phased array system, 2. In-house equipment, Radio Sci., 20, 1169-1176, 1985b.

Gonzales, C. A., M. C. Kelley, L. A. Carpenter, and R. H. Holzworth, Evidence for a magnetospheric effect on mid-latitude electric fields, J. Geophys. Res., 83, 4397-4399, 1978.

Kato, S., T. Ogawa, T. Tsuda, T. Sato, I. Kimura, and S. Fukao, The middle and upper atmosphere radar: First results using a partial system, Radio Sci., 19, 1475-1484, 1984.

Kato, S., T. Tsuda, M. Yamamoto, T. Sato, and S. Fukao, First results obtained with a middle and upper atmosphere (MU) radar, J. Atmos. Terr. Phys., 48, 1259-1267, 1986.

Oliver, W. L., S. Fukao, T. Sato, T. Tsuda, S. Kato, I.Kimura, A. Ito, T. Saryou, and T. Araki, Ionospheric incoherent scatter measurements with the MU radar: Observations during the large 
geomagnetic storm of 6-8 February 1986, submitted to J. Geophys. Res., 1988.

Richmond, A. D., S. MATSUShita, and J. D. TARPLEY, On the production mechanism of electric currents and fields in the ionosphere, J. Geophys. Res., 81, 547-555, 1976.

Richmond, A. D., M. Blanc, B. A. Emery, R. H. Wand, B. G. Fejer, R. F. Woodman, S. Ganguly, P. Amayenc, R. A. Behnke, C. Calderon, and J. V. Evans, An empirical model of quiet-day electric fields at middle and low latitudes, J. Geophys. Res., 85, 4658-4664, 1980.

Rishbeth, H., The F-layer dynamo, Planet Space Sci., 19, 263-267, 1971.

Rishbeth, H., Ion-drag effects in the thermosphere, J. Atmos. Terr. Phys., 41, 885-894, 1979.

Rishbeth, H., The F-region dynamo, J. Atmos. Terr. Phys., 43, 387-392, 1981.

Rishbeth, H. and O. K. Garriott, Introduction to Ionospheric Physics, 331 pp., Academic Press, New York, 1969.

Sato, T., A. Ito, W. Oliver, S. Fukao, T. Tsuda, and S. Kato, Ionospheric incoherent scatter measurements with the MU radar: Techniques and capability, submitted to Radio Sci., 1988.

TAYlor, G. N., Meridional F2-region plasma drifts at Malvern, J. Atmos. Terr. Phys., 36, 267-286, 1974. 